\title{
Delayed improvement in exercise capacity after cardioversion of atrial fibrillation to sinus rhythm
}

\author{
D P LIPKIN, M FRENNEAUX, R STEWART, J JOSHI, T LOWE, W J McKENNA \\ From the Cardiovascular Unit, Royal Postgraduate Medical School, Hammersmith Hospital, London
}

SUMMARY In some patients symptoms improve after the restoration of sinus rhythm from atrial fibrillation. To assess the size and mechanism of such change, exercise capacity and pulsed Doppler left ventricular inflow velocities were assessed in 20 patients with established atrial fibrillation. Treadmill exercise capacity was assessed by measuring maximal oxygen consumption and anaerobic threshold before and on day 1 and 28 days after elective DC cardioversion. The relative contribution of atrial contraction to left ventricular filling was determined by relating the maximum height of the $A$ wave to the maximum height of the $E$ wave $(A / E)$ of the Doppler velocity time curve. Cardioversion was successful in 14 patients. Maximal oxygen consumption and anaerobic threshold were unchanged on day 1 and increased by day 28 in all 14 patients. The percentage improvement was inversely related to the baseline values; however, the absolute improvement was small in all patients. The mean $A / E$ ratio increased significantly from day 1 to day 28 in all 14 patients.

Thus the restoration of sinus rhythm was associated with a delayed improvement in exercise capacity that may in part be due to a slow improvement in atrial contractility and peak cardiac output after cardioversion.

Direct current cardioversion of atrial fibrillation is often performed to improve cardiac output and exercise capacity by regularising the heart beat and restoring the atrial systolic contribution to ventricular filling. The magnitude and time course of this improvement are controversial and the relative contribution of atrial systole to stroke volume is still uncertain..$^{1-4}$ The purpose of the present study was to assess the mechanism and size of the increase in exercise capacity after cardioversion of atrial fibrillation to sinus rhythm.

\section{Patients and methods}

We studied 20 patients ( 18 men and 2 women; mean (SD) age 58(8) years). Atrial fibrillation had been present for 6-14 weeks and was associated with previous aortic or mitral valve surgery in five patients (performed 6-12 (mean 8) months before entry to the study), dilated cardiomyopathy in four patients,

Present address and requests for reprints to Dr D P Lipkin, Department of Cardiology, Royal Free Hospital, Pond Street, London NW3 6QG.

Accepted for publication 12 October 1987 coronary artery disease in seven patients, hypertrophic cardiomyopathy in two patients, and hypertension in one patient. Fibrillation was idiopathic in the remaining patient. The exercise tolerance of the patients was limited only by dyspnoea or fatigue. Patients were excluded if they had had a myocardial infarction during the previous six months, had evidence of myocardial ischaemia or ventricular tachycardia on exercise testing, or had chronic lung disease. There were four patients in New York Heart Association class I, 12 patients in class II, and four in class III. Resting left ventricular fractional shortening was assessed by $\mathbf{M}$ mode echocardiography recorded at the mitral valve leaflet tips (with cross sectional echocardiographic control) 24 hours and 28 days after cardioversion. In those patients in whom cardioversion failed left ventricular fractional shortening was assessed as the mean of 10 consecutive cardiac cycles. All echocardiographic and Doppler studies were performed by one observer. The reproducibility for left ventricular dimensions in our laboratory is within $5 \%$ of the previous value.

All patients were in controlled atrial fibrillation with a resting heart rate less than 70 beats/minute. Drug treatment which was stable for at least a month before entry to the study included digoxin (all 
patients (median dose $0.25 \mathrm{mg} /$ day), amiodarone (all patients (median dose $200 \mathrm{mg} /$ day), frusemide (10 patients (mean dose $55 \mathrm{mg} /$ day, range $40-80 \mathrm{mg} /$ day)), amiloride (eight patients (mean dose $8 \mathrm{mg} /$ day, range 5-10 $\mathrm{mg} /$ day)), bendroflurazide (three patients (dose of $5 \mathrm{mg} /$ day)), and captopril (one patient (dose $12.5 \mathrm{mg}$ three times a day). All patients were anticoagulated with warfarin for at least four weeks before cardioversion. Anaesthesia for DC cardioversion was induced with intravenous methohexitone (dose 1-2 $\mathrm{mg} / \mathbf{k g}$ ) injected over 30 seconds. Progressively increasing DC shocks of $100 \mathrm{~J}, 200 \mathrm{~J}$, and $320 \mathrm{~J}$ were used until the highest energy was reached or cardioversion was achieved.

Patients were exercised three hours after a light meal and at the same time of day, according to the Bruce protocol for those in New York Heart Association class I or II or a modified Naughton protocol with stages lasting 3 minutes for those in class III. ${ }^{5}$ The exercise protocol was not changed during the study. Patients were encouraged to exercise until they felt unable to continue; the test was stopped at the patient's request. Treadmill exercise capacity was assessed by measuring maximal oxygen consumption and anaerobic threshold with mass spectrometric analysis of mixed expired air before and on day 1 (24 hours) and day 28 after elective DC cardioversion. ${ }^{5}$ Patients repeated practice exercise tests before entry to the study until maximal oxygen consumption differed by less than $5 \%$ on two consecutive exercise tests. In the last 12 consecutive patients left ventricular inflow velocities were measured by pulsed Doppler during quiet respiration to evaluate the relative contribution of atrial contraction to left ventricular filling. A Doppler probe was placed at the cardiac apex with patients in the left lateral position and measurements were recorded during quiet respiration. The Doppler sample was placed in the region of the mitral leaflet tips, and the positioning of the sample was guided by cross sectional echocardiography. The sampling position was adjusted to obtain the peak inflow velocities by a combination of the spectral display and Doppler audio signal. The heights of the passive filling wave ( $E$ wave) and the atrial wave ( $A$ wave) were measured over 10 consecutive cycles to avoid sampling errors caused by minor beat to beat variations, and the mean values for the height of the $A$ wave, $E$ wave, and the $A / E$ ratio were calculated ${ }^{6}$ (fig 1). The reproducibility of Doppler inflow recording was determined in five normal subjects on two successive days by two observers. All drug treatment was unaltered for the duration of the study.

Results are quoted as mean (SE). Changes in exercise capacity, heart rate, and Doppler measurements after cardioversion were analysed by the Wilcoxon matched paired rank sum test. A p value $<0.05$ was considered to be significant.

\section{Results}

Sinus rhythm was restored in 14 of 20 patients by $1-3$
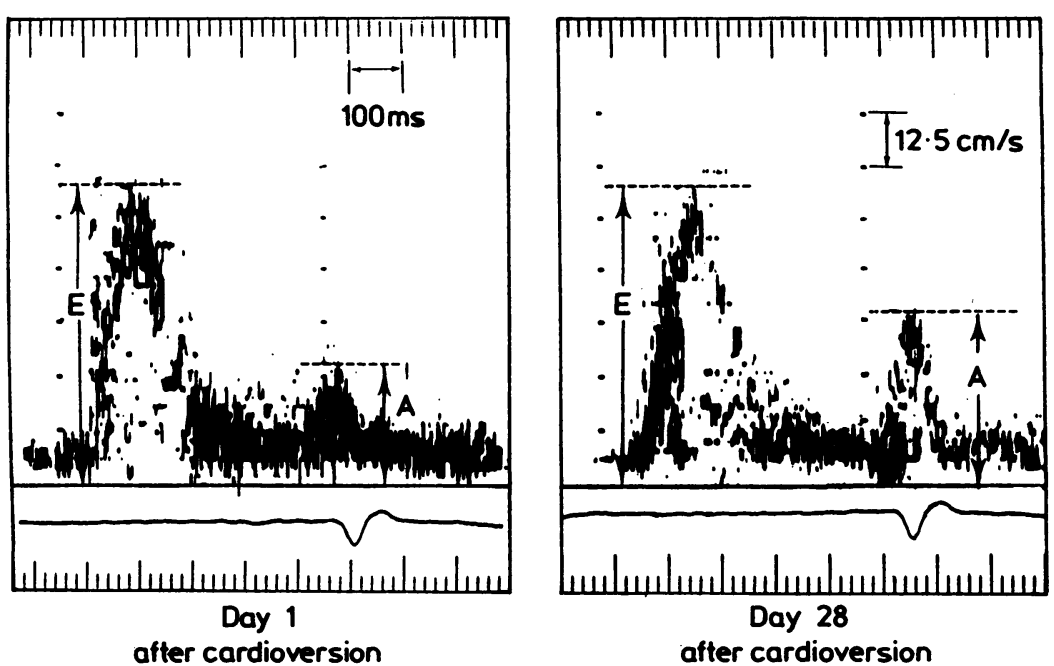

Fig 1 Peak left ventricular inflow velocities across the mitral valve in one patient on day 1 and day 28 after $D C$ cardioversion. The relative contribution of atrial contraction to left ventricular filling was determined by relating the mean height of the $A$ wave to the mean height of the $E$ wave of the velocity time curve during 10 consecutive cardiac cycles. 
Table 1 Changes in symptom limited maximum oxygen consumption, oxygen consumption at the anaerobic threshold, and heart rate before and day 1 and 28 after successful (patients 1-14) and unsuccessful (patients 15-20) DC cardioversion of atrial fibrillation to sinus rhythm

\begin{tabular}{|c|c|c|c|c|c|c|c|c|c|c|}
\hline \multirow[b]{2}{*}{$\begin{array}{l}\text { Case } \\
\text { No }\end{array}$} & & \multicolumn{3}{|c|}{$\begin{array}{l}\dot{V} \mathrm{O}_{2} \max \\
(\mathrm{ml} / \mathrm{kg} / \min )\end{array}$} & \multicolumn{3}{|c|}{$\begin{array}{l}\text { Anaerobic threshold } \\
\text { (ml/kg/min) }\end{array}$} & \multicolumn{3}{|c|}{$\begin{array}{l}\text { Peak exercise heart } \\
\text { rate (beats/min) }\end{array}$} \\
\hline & & $\begin{array}{l}\text { Day } \\
0\end{array}$ & 1 & 28 & $\underset{D}{\text { Day }}$ & 1 & 28 & $\begin{array}{l}\text { Day } \\
0\end{array}$ & 1 & 28 \\
\hline $\begin{array}{l}1 \\
2 \\
3 \\
4 \\
5 \\
6 \\
7 \\
8 \\
9 \\
10 \\
11 \\
12 \\
13 \\
14 \\
\text { Mean (SE) } \\
15 \\
16 \\
17 \\
18 \\
19 \\
20 \\
\text { Mean (SE) } \\
149(8 \cdot 6)\end{array}$ & $\begin{array}{l}\text { AVR } \\
\text { AVR } \\
\text { MVR } \\
\text { MVR } \\
\text { IDC } \\
\text { IDC } \\
\text { IDIO } \\
\text { H/T } \\
\text { HCM } \\
\text { IDC } \\
\text { CAD } \\
\text { CAD } \\
\text { CAD } \\
\text { CAD } \\
\text { MVR } \\
\text { IDC } \\
\text { HCM } \\
\text { CAD } \\
\text { CAD } \\
\text { CAD } \\
\end{array}$ & $\begin{array}{l}24 \\
16 \\
16 \\
31 \\
23 \\
11 \\
40 \\
15 \\
15 \\
19 \\
21 \\
24 \\
37 \\
24 \\
23(2) \\
31 \\
22 \\
21 \\
25 \\
21 \\
19\end{array}$ & $\begin{array}{l}24 \\
16 \\
15 \\
30 \\
23 \\
12 \\
40 \\
15 \\
16 \\
18 \\
22 \\
25 \\
38 \\
25 \\
23(2) \\
30 \\
23 \\
26 \\
22 \\
19 \\
23(2)\end{array}$ & $\begin{array}{l}27 \\
22 \\
23 \\
35 \\
24 \\
13 \\
43 \\
17 \\
18 \\
22 \\
24 \\
29 \\
38 \\
28 \\
26(2) \star \\
33 \\
21 \\
18 \\
25 \\
19 \\
18 \\
24(2)\end{array}$ & $\begin{array}{l}17 \\
13 \\
13 \\
25 \\
19 \\
11 \\
18 \\
14 \\
\text { NR } \\
17 \\
17 \\
19 \\
25 \\
18 \\
17(1) \\
20 \\
13 \\
20 \\
19 \\
18 \\
15 \\
22(3)\end{array}$ & $\begin{array}{l}17 \\
14 \\
13 \\
24 \\
19 \\
11 \\
19 \\
14 \\
\text { NR } \\
17 \\
18 \\
19 \\
25 \\
18 \\
18(1) \\
19 \\
14 \\
\text { NS } \\
19 \\
19 \\
13 \\
18(1)\end{array}$ & $\begin{array}{l}19 \\
20 \\
17 \\
30 \\
19 \\
12 \\
22 \\
15 \\
\text { NR } \\
20 \\
22 \\
23 \\
25 \\
19 \\
20(1) \dagger \\
20 \\
13 \\
17 \\
19 \\
17 \\
17 \\
17(1)\end{array}$ & $\begin{array}{l}150 \\
120 \\
170 \\
180 \\
190 \\
160 \\
214 \\
129 \\
180 \\
170 \\
150 \\
180 \\
195 \\
164 \\
168(7) \\
180 \\
150 \\
120 \\
130 \\
157 \\
153 \\
17(1)\end{array}$ & $\begin{array}{l}140 \\
115 \\
140 \\
155 \\
150 \\
105 \\
160 \\
101 \\
113 \\
130 \\
140 \\
166 \\
150 \\
129 \\
135(6) \ddagger \\
184 \\
155 \\
136 \\
153 \\
147 \\
125(24)\end{array}$ & $\begin{array}{l}140 \\
123 \\
150 \\
150 \\
152 \\
100 \\
150 \\
100 \\
115 \\
140 \\
145 \\
160 \\
155 \\
132 \\
137(5) \ddagger \\
185 \\
145 \\
125 \\
135 \\
144 \\
160 \\
155(8)\end{array}$ \\
\hline
\end{tabular}

$\dot{V}_{2}$ max, maximal oxygen consumption; AVR, after aortic valve replacement; MVR, after mitral valve replacement; IDC, idiopathic dilated cardiomyopathy; IDIO, idiopathic; H/T, hypertension; HCM, hypertrophic cardiomyopathy; CAD, coronary artery disease; NR, not reached.

* $p<0.002$ (compared with day 0 or 1 );

tp $<0.003$ (compared with day 0 or day 1 );

$\ddagger \mathrm{p}<0.001$ (compared with day 0 ).

(mean 1.2) DC shocks at a mean energy requirement of $130 \mathrm{~J}$. Functional class as assessed by the New York Heart Association did not alter during the study.

Maximal oxygen consumption and anaerobic threshold were unchanged at day 1 and rose significantly by $16(3 \%)$ and $18(14 \%)$ respectively on day 28 (p $<0.002,<$ p 0.003 respectively) (table 1 , figs 2 and 3). The absolute improvement in maximal oxygen consumption on day 28 was not related to left ventricular fractional shortening or to the exercise capacity (maximal oxygen consumption) before cardioversion. The percentage improvement in maximal oxygen consumption, however, correlated inversely with the value before cardioversion $(r=-0.57$, p < 0.01) (fig 4). Resting heart rates before cardioversion and on day 1 and 28 after cardioversion were 62(2), 69(2), and 70(3) beats/min. Peak exercise heart rate fell by day $1(p<0.001)$ but there was no significant difference between rates on days 1 and 28 after cardioversion (table 1 ).

In six patients cardioversion was unsuccessful. Maximal oxygen consumption and anaerobic threshold in these patients did not significantly alter during the study (table 1).

Mean left atrial size for all patients on entry to the 


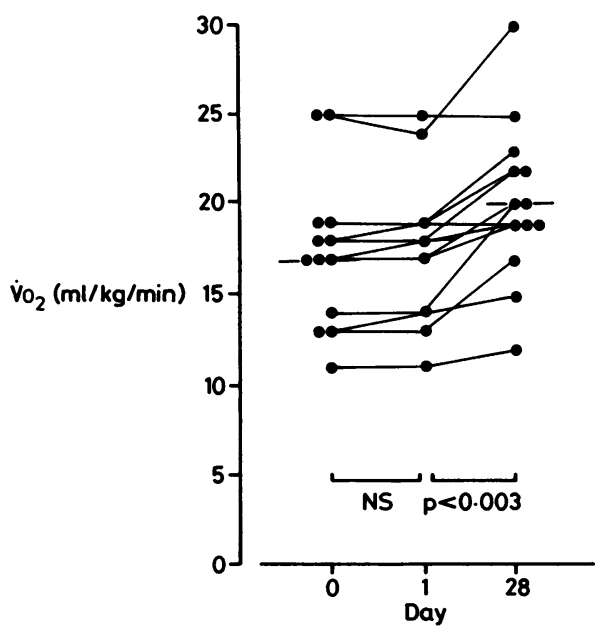

Fig 3 Change in oxygen consumption $\left(\dot{V}_{2}\right)$ at the anaerobic threshold before and on day 1 and 28 after DC cardioversion of atrial fibrillation to sinus rhythm.

study was $4.3(0.5) \mathrm{cm}$. The interobserver and intraobserver variability in normal subjects for the measurements of the height of the $\mathrm{A}$ and $\mathrm{E}$ wave and their ratio was $2-21 \%$ (mean 13\%), 2-36\%, (mean $15 \%$ ), and $4-35 \%$ (mean $17 \%$ ) respectively. Table 2 shows the Doppler results. During the Doppler studies the mean heart rates were similar on day 1 and day 28. In four patients no discernible $A$ wave was seen on visual inspection of the Doppler tracing on day 1 . Because velocities less than $12.5 \mathrm{~cm} / \mathrm{s}$ could not be measured accurately the height of the $A$ wave in these patients was assumed to be $12.5 \mathrm{~cm} / \mathrm{s}$. The mean $A / E$ ratio was 0.32 (range $0.15-0.60$ ) on day 1 and increased significantly in all patients to 0.80 (range $0.27-1.44$ ) by day 28 (p < 0.003) (fig 5). The increase in the $A / E$ ratio resulted solely from an increase in the size of the atrial wave (table 2). Left

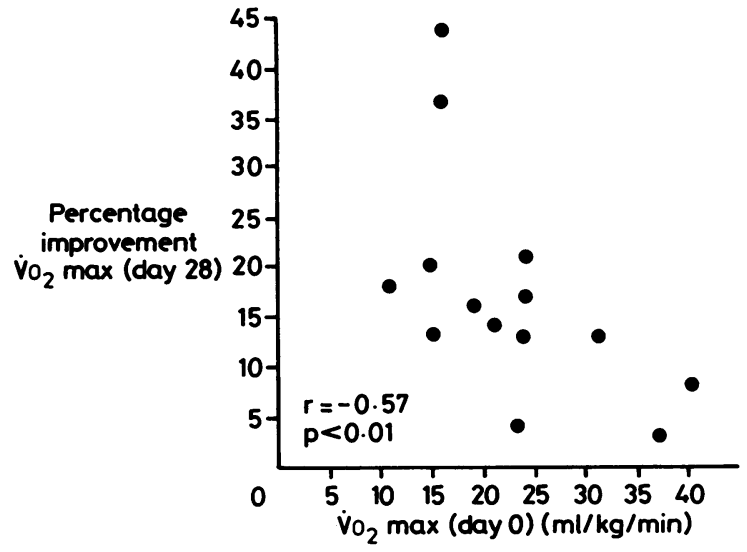

Fig 4 Percentage improvement in symptom limited maximal oxygen consumption ( $\mathrm{V}_{2}$ max) related to maximal oxygen consumption before cardioversion (day 0 ).

ventricular fractional shortening was similar in patients who underwent successful and unsuccessful cardioversion $(23(7) \%$ and $27(2) \%$ respectively). There was no significant change in left ventricular fractional shortening between day 1 and 28 in patients in whom cardioversion was successful (23(5)\% vs $22(5) \%$ respectively).

\section{Discussion}

Several haemodynamic studies have demonstrated an increase in cardiac output ${ }^{3}{ }^{7-10}$ but to our knowledge this is the first study to measure the size of the increase in exercise capacity after conversion of atrial fibrillation to sinus rhythm. Assessment of changes in respiratory gas exchange on exercise enabled us objectively to assess exercise capacity. ${ }^{5}$ The results show that symptom limited maximum oxygen consumption and anaerobic threshold do not rise 24 hours after DC cardioversion, but a mean

Table 2 Measurement of the height of the $A$ wave, $E$ wave, and $A / E$ ratio on day 1 and 28 after successful DC cardioversion of atrial fibrillation to sinus rhythm. Doppler recordings were made at similar RR intervals on day 1 and 28

\begin{tabular}{|c|c|c|c|c|c|c|c|c|}
\hline \multirow{2}{*}{$\begin{array}{l}\text { Case } \\
\text { No }\end{array}$} & \multicolumn{2}{|c|}{ A wave $(\mathrm{cm} / \mathrm{s})$} & \multicolumn{2}{|c|}{ E rove $(\mathrm{cm} / \mathrm{s})$} & \multicolumn{2}{|c|}{ ECG RR interval } & \multicolumn{2}{|l|}{$A / E$ ratio } \\
\hline & Day 1 & Day 28 & Day 1 & Day 28 & Day 1 & Day 28 & Day 1 & Day 28 \\
\hline $\begin{array}{l}3 \\
4 \\
5 \\
6 \\
7 \\
8 \\
9 \\
10 \\
11 \\
12 \\
13 \\
14 \\
\text { Mean (SE) } \\
\text { p }\end{array}$ & $\begin{array}{l}12 \cdot 5 \\
35 \cdot 0 \\
36 \cdot 2 \\
12.5 \\
18 \cdot 7 \\
12.5 \\
36 \cdot 0 \\
19 \cdot 4 \\
27 \cdot 0 \\
12 \cdot 5 \\
31 \cdot 2 \\
16 \cdot 0 \\
22 \cdot 5(2 \cdot 9) \\
\end{array}$ & $\begin{array}{l}33 \cdot 4 \\
93 \cdot 0 \\
72 \cdot 0 \\
30 \cdot 0 \\
43 \cdot 7 \\
23 \cdot 4 \\
90 \cdot 0 \\
82 \cdot 0 \\
76 \cdot 4 \\
23 \cdot 2 \\
36 \cdot 9 \\
25 \cdot 1 \\
52 \cdot 4(8 \cdot 0) \\
3\end{array}$ & $\begin{array}{c}39 \cdot 0 \\
116.0 \\
112 \cdot 5 \\
54 \cdot 3 \\
47 \cdot 5 \\
73 \cdot 5 \\
97 \cdot 3 \\
59 \cdot 0 \\
77 \cdot 1 \\
83 \cdot 3 \\
51 \cdot 2 \\
44 \cdot 4 \\
71 \cdot 3(7 \cdot 7)\end{array}$ & $\begin{array}{r}35.9 \\
125.0 \\
62.0 \\
72.5 \\
63.7 \\
68.7 \\
62.5 \\
57.0 \\
84.0 \\
86.0 \\
52.0 \\
50 \cdot 2 \\
68 \cdot 2(6.5)\end{array}$ & $\begin{array}{l}850 \\
870 \\
850 \\
1040 \\
1100 \\
1080 \\
730 \\
770 \\
1070 \\
920 \\
840 \\
860 \\
915(36)\end{array}$ & $\begin{array}{l}890 \\
800 \\
890 \\
1000 \\
1320 \\
1340 \\
660 \\
770 \\
1210 \\
940 \\
800 \\
890 \\
959(63)\end{array}$ & $\begin{array}{l}0.32 \\
0.30 \\
0.32 \\
0 \cdot 23 \\
0 \cdot 39 \\
0 \cdot 17 \\
0 \cdot 37 \\
0.33 \\
0.35 \\
0 \cdot 15 \\
0.60 \\
0.36 \\
0.32(0.03)\end{array}$ & $\begin{array}{l}0.93 \\
0.75 \\
1.16 \\
0.41 \\
0.68 \\
0.34 \\
1.44 \\
1.44 \\
0.91 \\
0.27 \\
0.71 \\
0.50 \\
0.80(0.12) \\
3\end{array}$ \\
\hline
\end{tabular}




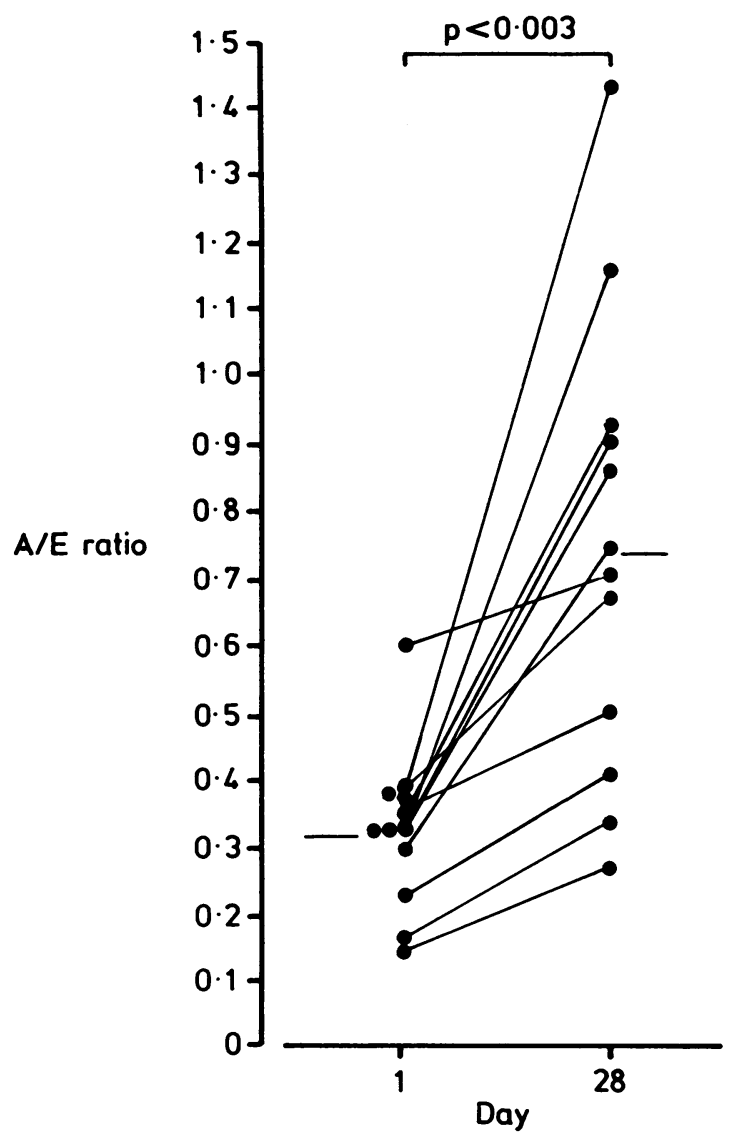

Fig 5 Change of $A / E$ ratio from day 1 to day 28 after $D C$ cardioversion (10 patients)

increase of $16 \%$ and $18 \%$ respectively is seen 28 days later. The nature of the study prevented a placebo blind trial. It is unlikely that greater encouragement by the observing doctor during the exercise tests performed after cardioversion, or increased patient familiarity with treadmill exercise, were responsible for the improvement. This is because we took care to ensure that all patients had practised on the treadmill and that their maximal oxygen consumption did not differ by $>5 \%$ on two successive tests before entry to the study. The exercise tests before and after cardioversion were performed in most instances by different doctors who were unaware of the previous exercise data. Changes in maximal oxygen consumption were associated with a corresponding change in anaerobic threshold, suggesting a true change in exercise capacity rather than an alteration in patient motivation..$^{5}$ Also the maximal oxygen consumption and anaerobic threshold were unchanged at day 28 in those patients in whom cardioversion failed.

Failure to demonstrate an improvement in exercise capacity one day after DC cardioversion could have been influenced by the after effects of general anaesthesia and of the DC shock itself. Though these possibilities cannot be excluded they are unlikely to explain the observed results. Firstly, anaesthesia lasted for less than 20 minutes and the first exercise test was performed at least $\mathbf{2 4}$ hours after cardioversion. Secondly, important cardiac damage, as detected by a rise in the cardiac isoenzyme of creatine kinase, did not occur with repeated elective DC shocks. " Thirdly, there was no difference in left ventricular fractional shortening between day 1 and 28 in the patients who underwent successful DC cardioversion in the present study. Finally, maximal oxygen consumption did not increase between day 1 and 28 in the patients in whom sinus rhythm was not restored by cardioversion.

The mechanism of the delayed improvement in exercise capacity is of interest. The reported effects of cardioversion of atrial fibrillation to sinus rhythm on haemodynamic function are inconsistent..$^{7-1012}$ This may be because of variation in the timing and method of assessment of atrial function after cardioversion. ${ }^{12-16}$

We have looked in a novel way at the contribution of atrial contraction to left ventricular filling by relating the height of the $A$ wave to the height of the $E$ wave in the Doppler velocity time curve of flow across the mitral valve. The height of the $A$ wave in the Doppler tracing is dependent on the pressure gradient across the mitral valve at end diastole. Because a major change in left ventricular compliance in individual patients during the study is unlikely, the height of the $A$ wave may be taken to relate to the force of atrial contraction. The A wave was absent or small immediately after cardioversion and increased significantly at one month. In the present study peak exercise heart rates at day 1 and 28 were similar, suggesting that the improvement in exercise capacity was the result of an improvement in stroke volume that followed an increased atrial contribution to left ventricular filling.

There is an alternative explanation for the late improvement in exercise capacity after cardioversion. Cardiac output improves immediately after cardioversion because of the regularisation of rhythm that permits improved ventricular filling, but there is delayed improvement in factors which limit blood flow to the skeletal muscle. It is now firmly established that inotropic or vasodilator treatment in chronic heart failure improves cardiac output immediately, but without a corresponding improvement in exercise capacity. ${ }^{17}$ The improvement in exercise capacity that is seen with long term 
treatment with angiotension converting enzyme inhibitors is limited to those patients in whom skeletal muscle blood flow increases. ${ }^{18} 19$

Conversion of atrial fibrillation to sinus rhythm was associated with a small (less than 1 metabolic equivalent) delayed improvement in exericse capacity. The greatest effect was seen in those patients with more severe exercise limitation. Slow return of atrial contractility and inhibition of factors limiting skeletal muscle blood flow may be responsible for the late benefit.

\section{References}

1 Morris JJ, Entman M, North WC, Kong Y, McIntosh $H$. The changes in cardiac output with reversion of atrial fibrillation to sinus rhythm. Circulation 1965; 31:670-8.

2 Reale A. Acute effects of counter shock conversion of atrial fibrillation upon right and left heart haemodynamics. Circulation 1965;32:214-22.

3 Rowlands DJ, Logan WF, Howitt G. Atrial function after cardioversion. Am Heart J 1967;74:149-60.

4 Kaplan MA, Gray RE, Iseri LT. Metabolic and hemodynamic responses to exercise during atrial fibrillation and sinus rhythm. Am J Cardiol 1968; 22:543-9.

5 Lipkin DP, Perrins J, Poole-Wilson PA. Respiratory gas exchange in the assessment of patients with impaired ventricular function. $\mathrm{Br}$ Heart $J$ 1985;54:321-8.

6 Rokey R, Kuo LC, Zoghbi WA, Limacher M, Quinones MA. Determination of parameters of left ventricular diastolic filling with pulsed Doppler echocardiography: comparison with angiography. Circulation 1985;71:543-50.

7 Shapiro $\mathbb{W}$, Klein G. Alterations in cardiac function immediately following electoral conversion of atrial fibrillation to normal sinus rhythm. Circulation 1968;38:1074-84.
8 Resnekov L. Haemodynamic studies before and after electrical conversion of atrial fibrillation and flutter to sinus rhythm. Br Heart J 1967;29:700-8.

9 Resnekov L, McDonald L. Electroversion of lone atrial fibrillation and flutter including haemodynamic studies at rest and on exercise. Br Heart J 1971; 33:339-50.

10 Orlando JR, Van Herick R, Aronow WS, Olson HG. Hemodynamics and echocardiograms before and after cardioversion of atrial fibrillation to normal sinus rhythm. Chest 1979;76:521-6.

11 Ehsani A, Ewy GA, Sobel BE. Effect of counter-shock on serum creatine phosphokinase (CPK) isoenzyme activity. Am J Cardiol 1976;37:12-8.

12 Grover DN, Mathur VS, Shrivastava S, Roy SB. Electromechanical correlation of left atrial function after cardioversion. Br Heart J 1971;33:226-32.

13 Mahlich J, Schweizer W, Burkart F. Atrial function after cardioversion for atrial fibrillation. Br Heart $J$ 1973;35:24-7.

14 Ikram H, Nixon PGF, Arcan T. Left atrial function after electrical conversion to sinus rhythm. Br Heart J 1968;30:80-3.

15 Macieira-Coelho E, Alves MG, Almiro H, Granate J, Coelho E. Atrial activity following conversion of experimental atrial fibrillation by direct current shock. Acta Cardiol (Brux) 1968;23:439-53.

16 Logan WFWE, Rowlands DJ, Howitt G, Holmes AM. Left atrial activity following cardioversion. Lancet 1965;ii:471-3.

17 Lipkin DP, Poole-Wilson PA. Symptoms limiting exercise tolerance in chronic heart failure. $\mathrm{Br} \mathrm{Med} \mathrm{J}$ 1986;292:1030-1.

18 Lipkin DP, Poole-Wilson PA. Treatment of chronic heart failure-review of recent drug trials. $\mathrm{Br} \mathrm{Med} \mathrm{J}$ 1985;291:993-6.

19 Mancini D, Davis L, Wexler JP, Chadwick B, LeJemtel TH. Long-term therapy with captopril improves maximal oxygen uptake by increasing skeletal muscle blood flow in patients with heart failure [Abstract]. Circulation 1984;70:193A. 